\title{
WHO Digital Intelligence Analysis for Tracking Narratives and Information Voids in the COVID-19 Infodemic
}

\author{
Tina D PURNAT ${ }^{\mathrm{a}, 1}$, Paolo VACCA ${ }^{\mathrm{b}}$, Stefano BURZO ${ }^{\mathrm{a}}$, Tim ZECCHIN $^{\mathrm{b}}$, Amy \\ WRIGHT $^{\mathrm{b}}$, Sylvie BRIAND ${ }^{\mathrm{c}}$ and Tim NGUYEN ${ }^{\mathrm{c}}$ \\ ${ }^{a}$ Department of Digital Health and Innovation, Science Division, World Health \\ Organization, Geneva, Switzerland \\ ${ }^{\mathrm{b}}$ Media Measurement LTD, London, United Kingdom \\ ${ }^{c}$ Department of Global Infectious Hazards Preparedness, Emergency \\ Preparedness, World Health Organization, Geneva, Switzerland
}

\begin{abstract}
The COVID-19 pandemic is the first to unfold in the highly digitalized society of the 21 st century and is therefore the first pandemic to benefit from and be threatened by a thriving real-time digital information ecosystem. For this reason, the response to the infodemic required development of a public health social listening taxonomy, a structure that can simplify the chaotic information ecosystem to enable an adaptable monitoring infrastructure that detects signals of fertile ground for misinformation and guides trusted sources of verified information to fill in information voids in a timely manner. A weekly analysis of public online conversations since 23 March 2020 has enabled the quantification of running shifts of public interest in public health-related topics concerning the pandemic and has demonstrated the frequent resumption of information voids relevant for public health interventions and risk communication in an emergency response setting.
\end{abstract}

Keywords. Infodemic, infodemic management, social listening, social monitoring, social media, pandemic preparedness, pandemic response, risk communication

\section{Introduction}

Since the beginning of the COVID-19 pandemic, digital communications and social networking have supported the rapid growth of real-time information sharing about the virus and the disease in the public domain. The breadth of the conversation, the diversity of sources and polarity of opinions have resulted in a sometimes indiscriminate amplification of verified and unverified information alike. This infodemic [1] (too much information including false or misleading information in digital and physical environments during an acute public health event, which leads to confusion, risk-taking and behaviors that can harm health, and lead to mistrust in health authorities and public health response) can elongate or amplify outbreaks, and can reduce effectiveness of pandemic response efforts and interventions.

\footnotetext{
${ }^{1}$ Tina D Purnat, corresponding author, Department of Digital Health and Innovation, Science Division,
} World Health Organization, Geneva, Switzerland; E-mail: tina.purnat@gmail.com. 
To address this challenge, the WHO Information Network for Epidemics (EPI-WIN), in collaboration with research partners, developed a public health infodemic intelligence analysis methodology for weekly analysis of digital media data to identify, categorize, and understand the key concerns expressed in online conversations [2]. The application of this methodology produced infodemic digital intelligence, providing WHO with week-on-week information for prioritization of actions to fill information voids with high-quality health information. Where there is lack of quality information about topics that users online are concerned about, such topics can fast become filled with conjecture, speculation, low-quality health information, and viral misleading content [3], thus potentially causing harm to communities. EPI-WIN's approach therefore focuses on infodemic signals detection - identifying or predicting rising areas of concern and information voids in the online information eco-system and provide immediately actionable intelligence for input into decision-making and risk communication $[4,5]$, to complement rumor tracking activities and provide the right health information at the right time in the right format.

\section{Methods}

\subsection{Data sources}

The analysis was based on the aggregation of publicly available social and news media and online search data in English and French by using Meltwater Explore [6] and Google Trends. The platform drew verbatim mentions of keywords associated with pre-defined topics from ten open data sources and fora (Twitter, Blogs, Facebook, Reddit, Forums, Comments, Instagram, Product Reviews, YouTube).

Thirty-five keyword-based searches (per language) were created and grouped according to a set of pandemic public health taxonomy categories developed specifically for this analysis. The taxonomy has four thematic categories of conversation about COVID-19 and public health response: (1) the cause of the illness - How did the virus emerge and how is it spreading?; (2) the illness - What do we know about the virus, what are the symptoms and how is it transmitted?; (3) the treatment - How can it be cured?; and (4) the interventions - What is being done by authorities and institutions? In addition, a fifth area was designed to look at public perceptions on circulating information, i.e. meta-conversations about evidence and statistics, mis- and dis-information, successful and harmful content, or key influencers that have been actively amplifying information on COVID-19. Each of the 35 taxonomy categories encompassed a list of topics that capture different aspects of that segment of the online conversation on COVID-19.

Identification of information voids was done on three key parameters within the weekly dataset: the volume (i.e., how many social media items referred to topic X?), the velocity (i.e., the rate of increase of the number of social media items that have engaged with topic $\mathrm{X}$ over the course of past week), and the emotion (i.e., has topic X triggered a significant surge in language that expresses denial, sadness, anxiety, fear, anger, or acceptance?). The volume was the sum of the online items that mentioned COVID-19 together with a keyword related to each tracked topic. Velocity equaled the weekly percentage increase of the volume of media items aggregated under each topic and taxonomy. Emotion analysis with 210 search strings quantified the proportion of media items that contained at least one mention of COVID-19, at least one mention of a topicrelated keyword, and at least one mention of any tracked keyword associated with each 
emotion (e.g. swearwords expressing anger). Questions were detected by pairing each of the 35 topic search strings with additional Boolean strings that enabled the isolation of subsets that, within each topical conversation featured English and French question syntax (e.g. question words, verb-subject inversion, auxiliaries).

Sentiment monitoring solutions are available on most advanced media analytics tools, but these approaches benefit primarily brand performance analysis and provide less accurate results for the type of analysis used here [7,8]. Analysis therefore focused on "emotion" rather than sentiment. The categorization of emotions was based on existing literature on the stages of grief in the context of a pandemic [9,10]: denial, sadness, anxiety, fear, anger, and acceptance. Analysis described how each emotion has played out in the conversation on COVID-19 and helped to isolate those conversations that have generated significant bursts of emotional response, thus suggesting the potential requirement for consideration.

\subsection{Analysis method}

Since late March 2020, 44 weekly global analysis reports were produced supplying the EPI-WIN team and its partners with early warnings of the points of concern expressed by online users $[2,4]$. By 29 Jan 2021, the data sample consisted of 640 million from about a total of 1.13 billion public social media posts in English or French mentioning COVID-19 (nearly 4\% of all public social media conversation produced in English and French globally since March 2020).

Each week, social media conversations were segmented based on levels of velocity and quantitatively examined for public engagement (e.g., likes, shares, poll votes, reactions), hashtags, and most used keywords and phrases. A qualitative analysis of narratives, influencers, and public reactions as reflected in comment threads was also done. This identified how the public was more likely to understand and explain aspects of the pandemic based not only on the prominence of these narratives in the overall weekly digital conversation (rank of category by volume), but especially on the velocity whereby these aspects have attracted interest in the preceding seven days (rank of category by velocity and its week-on-week change, as well as the amplification of these sub-sections of the conversation.

A qualitative analysis of interlinks between topics and their disappearance and resumption over a long period of time informed key weekly insights about the context within which these narratives developed. The qualitative analysis prioritized the flagging of widespread confusion or frequently asked questions, the rapid amplification of misinformation, or ad-hoc aspects of the conversation that are particularly relevant to public health, such as vaccine hesitancy ahead of, and during a vaccination campaign. The identified areas of concern on social media were further evaluated using engagement data and Google search trends to determine whether a significant number of online users have been looking for information on these topics.

\section{Results}

The narratives and questions in the online conversations shifted as the pandemic evolved over the course of 2020. Based on the average weekly rises of the topics within each of the five taxonomy categories in four 11-week periods between 23 March 2020 and 27 January 2021, it was observed that Periods 1 and 2 were characterized by a steady 
increase in conversations about the interventions. Whilst interest in the illness decreased in Period 1, it increased in the second period. In Period 3, the treatment had the highest velocity in digital conversations, and in Period 4 the public attention returned towards the interventions.

At the same time, during the 44-week period of analysis, topics reoccurred periodically. All 35 categories of topics that were tracked resumed their velocity throughout the reporting period for an average of 18 weeks combined. The two most recurring topics to attract increasing interest were Vaccines and Asymptomatic transmission (rising for 22 weeks) followed by Immunity, the Cause of the virus, Vulnerable communities and Reduction of movement (21 weeks), and Risk factors based on demographics and risk of misinformation (20 weeks). Digital conversations on the cause of the virus, misinformation as a phenomenon, and immunity had the longest continuous periods of growing velocity: the conversation on the cause has grown both in the first half and second half of the analysis period for six continuous weeks whilst meta-conversation about misinformation has grown for six consecutive weeks during the first half of the reporting period. Immunity has grown for five consecutive weeks both in June-July 2020 and at the very end 2020, between November and December 2020.

Overlaps of conversations between several topic categories (for example, where conversations discussed both transmission settings and faith) have been observed consistently throughout the analysis period. This indicates that the tracked conversation topics are deeply intertwined.

The taxonomy has required review and maintenance. At the beginning of summer 2020, the taxonomy was reviewed and minor amendments were done to ensure the analysis could detect conversation on new concepts that had entered public discourse (e.g. airborne transmission, mutation) as well reflect the way the digital public referred to the virus (for instance, a switch was noticed from "COVID-19", "coronavirus" or "Sars-Cov2" to simply "covid" or "corona").

\section{Discussion}

The application of this taxonomy to online social listening week-on-week has resulted in a better in-time understanding of the evolution and dynamics of high velocity conversations about COVID-19 globally during the pandemic and proposes a quantifiable approach to support planning of risk communication response. For example, monitoring and characterizing reoccurring topics can guide reevaluation and updating of risk communication messages and materials produced previously. In addition, narrative overlaps across categories have better informed the qualitative insights on the scope of the narratives that have attracted most engagement from the digital public.

A limitation of global conversation analysis is that only major online narrative themes and information voids are identified. Smaller emerging conversations may not be picked up in analysis in a timely manner. It has also been observed that the global English-language dataset is prone to overrepresent the voice of social media geographies or communities that are more digitally proactive than others. A key challenge was the digital amplification of discourse pertaining to US politics, the elections and the digital prominence of the US public thereof [11]. In such situations, exclusion keywords should be used to exclude major events or large-scale media coverage from analysis, so that they do not mask narratives that are relevant for public health authorities. Future work should study how the results differ in different linguistic communities and the effect of 
geographies that may be super influencers. Lastly, known limitations are proportional representation of data collected by the content aggregator and over-representation of Twitter content [12]. Further streamlining of the methods and tools, and evaluation of impact risk communication interventions based on identified information voids is needed.

\section{Conclusions}

Applying this methodology to a public health context has provided invaluable insight, inspiring new ways of thinking and communicating risk during health emergencies. This research framework is now being used in Canada by the National Institute of Public Health of Québec (INSPQ) as an input into public health communication strategies in the province, and is piloted at country level in WHO Regional Offices for South East Asia, Western Pacific and Africa. With the expansion of the methodology across regions, there is now an opportunity to compare the similarities and differences of how this insight can be used at the local level, and add the layer of multilingual content analysis.

\section{References}

[1] Tangcharoensathien V, Calleja N, Nguyen T, Purnat T, D’Agostino M, Garcia-Saiso S, Landry M, Rashidian A, Hamilton C, AbdAllah A, Ghiga I. Framework for managing the COVID-19 infodemic: methods and results of an online, crowdsourced WHO technical consultation. Journal of Medical Internet Research. 2020;22(6):e19659.

[2] Coronavirus disease 2019 (COVID-19) Situation Report 100. 29 April 2020 World Health Organization. Available at: https://www.who.int/docs/default-source/coronaviruse/situation-reports/20200429-sitrep100-covid-19.pdf?sfvrsn=bbfbf3d1 6, Accessed January 29, 2021.

[3] Shane T and Noel P. Data deficits: why we need to monitor the demand and supply of information in real time. 28 Sep 2020 Available at: https://firstdraftnews.org/long-form-article/data-deficits/, Accessed January 29, 2021

[4] Coronavirus disease 2019 (COVID-19) Situation Report 128. 27 May 2020 World Health Organization. Available at: https://www.who.int/docs/default-source/coronaviruse/situation-reports/20200527-covid19-sitrep-128.pdf?sfvrsn=11720c0a_2, Accessed_January 29, 2021.

[5] Zecchin, T. Web/Social media listening and analytics for COVID-19 communication WHO ad hoc consultation on managing the COVID-19 infodemic. Available at: https:/www.who.int/teams/riskcommunication/infodemic-management/who-ad-hoc-online-consultation-on-managing-the-covid-19infodemic, Accessed January 29, 2021.

[6] Rowland, L. What are the Data Sources in Explore? Meltwater, Available at: https://help.meltwater.com/en/articles/4064549-what-are-the-data-sources-in-explore, Accessed March 5,2021 .

[7] Kenyon-Dean K., Ahmed E., Fujimoto S, Georges-Filteau J, Glasz C, Kaur B, Laland A, Bhanderi S, Belfer R, Kanagasabai N, Sarrazingendron R, Verma R, Ruths D. Sentiment Analysis: It's Complicated! Proceedings of NAACL-HLT; 2018 Jun 1-6; New Orleans, Louisiana: Association for Computational Linguistics, p. 1886-1895.

[8] Kirilenko AP, Stepchenkova SO, Kim H. Automated Sentiment Analysis in Tourism: Comparison of Approaches. Journal of Travel Research. 2018 Nov; 57(8):999-1011

[9] Kübler-Ross E, Wessler E, Avioli LV. On death and dying. Jama 1972 Jul;221(2)-174-179

[10] Aiello LM, Quercia D, Zhou K, Constantinides M, Ścepanović S, Joglekar S (2020) How epidemic psychology works on social media: evolution of responses to the covid-19 pandemic. arXiv preprint arXiv;2007.

[11] Samoilenko SA, Miroshnichenko A. Profiting From the "Trump Bump": The Effects of Selling Negativity in the Media. In: Handbook of Research on Deception, Fake News, and Misinformation Online. IGI Global; 2019. p. 375-391.

[12] Hayes JL, Britt BC, Evans W, Rush SW, Towery NA, Adamson AC. Can Social Media Listening Platforms' Artificial Intelligence Be Trusted? Examining the Accuracy of Crimson Hexagon's (Now Brandwatch Consumer Research's) AI-Driven Analyses. Journal of Advertising. 2020 Sep; 50(1):81-91. 